\title{
Role of the Glucagon-like Peptide-1 Receptor Agonist in Maintaining Pluripotency in Human Embryonic Stem Cells
}

\author{
Methichit Chayosumrit, ${ }^{*}$, Anandwardhan A. Hardikar² and Kuldip S. Sidhu ${ }^{1}$ \\ ${ }^{I}$ Stem Cell Laboratory, School of Psychiatry, Faculty of Medicine, University of New South Wales, NSW, Australia \\ ${ }^{2}$ Diabetes and Pancreas Biology Section, The O'Brien Institute, St Vincent's Hospital, The University of Melbourne, \\ Melbourne, VIC, Australia
}

\begin{abstract}
Previous studies have demonstrated that glucagon-like peptide-1 (GLP-1) stimulates $\beta$-cell formation and insulin secretion. Currently, there has been no report in understanding the effect of GLP-1 / its agonist exendin- 4 in differentiation of human embryonic stem cells (hESCs) to definitive endodermal (DE). We hypothesized that exendin-4 signaling in hESCs via GLP-1 receptor (GLP-1R) may have potential role in DE differentiation. The effect of Ex-4 on pluripotent hESCs and the combined effect of Ex-4 and activin A-treated hESC-derived DE were examined. Analysis by quantitative real-time PCR (qPCR) demonstrates that Ex-4 alone was not sufficient to enhance DE formation in hESCs. On the other hand, a combinatorial treatment with activin A and Ex-4 resulted in significant decrease in expression levels of DE markers. The miRNA expression profiles between activin A-treated hESCs and activin A/Ex-4-treated hESCs after 5 days of treatment demonstrated similar expression levels of endoderm and pancreas-associated miRNAs. However, it was shown that the levels of pluripotency-associated miRNAs, miR-302a* and miR302c*, were upregulated in the presence of Ex-4. Furthermore, it was observed that exposure to bFGF and Ex-4 in apoptosis-inducing medium resulted in downregulation of CASP3 and p53. Taken together, these data revealed the possibility of Ex-4 in maintaining pluripotency and inhibiting apoptosis. The knowledge of GLP-1 signaling pathways could be useful for understanding the mechanism of GLP-1R-ligand interactions and their relevance to hESC development.
\end{abstract}

Keywords: Human embryonic stem cells, glucagon-like peptide-1, differentiation, definitive endoderm.

\section{INTRODUCTION}

GLP-1 is an incretin hormone encoded by the glucagon gene and is secreted in response to ingested food [1]. GLP-1 signaling involves the formation of new $\beta$-cells through enhanced proliferation of existing $\beta$-cells, induction of islet neogenesis $[2,3]$ as well as an inhibition of $\beta$-cell apoptosis $[4,5]$. GLP-1 is sensitive to the enzyme dipeptidyl peptidase IV (DPPIV) and therefore, it has a short half life in the body. The long acting agonist, Ex-4, which is resistant to DPPIV, is being used to replace GLP-1 in most studies [2, 3, 6, 7]. A previous study demonstrated that GLP-1 reduced DNA fragmentation and improved cell survival in freshly isolated human islets [4]. GLP-1 increased the expression of antiapoptotic proteins $B C L-2$ and $B C L-X L$, as well as decreased expression of CASP3, CASP8, CASP9 [5]. Although the GLP-1R is predominantly localized to pancreatic islets, a study has demonstrated that it is also expressed in different human tissues including lung, brain, kidney, stomach and heart [8]. Cellular signaling pathways of GLP-1 were widely examined in pancreatic islets. However, less has been elucidated in neural or ESCs. There are a few reports on GLP-1 analogues acting as a neuronal protective agent in preventing cell death induced by amyloid $\beta$ peptide $\left(\mathrm{A} \beta_{1-42}\right)$,

*Address correspondence to this author at the Stem Cell Laboratory, School of Psychiatry, Faculty of Medicine, University of New South Wales, NSW, Australia; Tel: +612 9385 2002; Fax: +612 9385 3938;

E-mail: methichit@gmail.com oxidative stress and membrane lipid peroxidation caused by iron in cultured hippocampus neurons $[9,10]$. Ex-4 was also investigated in Parkinson's disease (PD) as a protective agent against cytokine mediated apoptosis [11]. These data suggested that GLP-1/Ex-4 plays an important role in preventing apoptosis in various cell types. However, it remains unknown if GLP-1R-ligand regulates pluripotency and/or apoptosis in hESCs. Here, we demonstrate that GLP$1 \mathrm{R}$ ligands are involved in maintenance of pluripotency as well as inhibition of apoptosis in hESCs. These studies indicate that GLP-1R mediated signaling in hESCs may influence cellular viability in vitro or upon transplantation, in vivo.

\section{MATERIALS AND METHODOLOGY}

\section{Culture of hESCs on Feeder Layer}

The hESC line used in this study was hES3 cells. Undifferentiated hESCs were maintained on $\gamma$-irradiated (45 Gy) human fetal fibroblasts (HFFs) and cultured in serum replacement (SR) medium, which contains Knockout Dulbecco's modified Eagle's medium (KODMEM)- high glucose, $20 \%$ knockout serum replacer, $2 \mathrm{mM}$ L-glutamine, $0.1 \mathrm{mM}$ non-essential amino acids, $0.1 \mathrm{mM} \mathrm{2-mercapto-}$ ethanol, $1 \mathrm{X}$ insulin-transferrin-selenium, $25 \mathrm{U} / \mathrm{mL}$ penicillin, $25 \mu \mathrm{g} / \mathrm{mL}$ streptomycin and $4 \mathrm{ng} / \mathrm{mL}$ basic fibroblast growth factor (bFGF). hESCs were subcultured approximately once every 5 days by incubation with $0.05 \%$ Trypsin for $2 \mathrm{~min}$ as 
described previously [12]. Primary HFFs were cultured in FDMEM consisting of DMEM-high glucose containing 10\% fetal bovine serum (FBS), $25 \mathrm{U} / \mathrm{mL}$ penicillin and $25 \mu \mathrm{g} / \mathrm{mL}$ streptomycin (all reagents were from Invitrogen, Carlsbad, CA).

\section{Differentiation of hESCs to Definitive Endoderm Cells}

To examine the effect of Ex-4 on hESCs and activin Atreated hESCs, cells were diffierentiated to DE using a previously published protocol, which used $100 \mathrm{ng} / \mathrm{mL}$ activin A (R\&D systems, Inc. Minneapolis, Minnesota, USA) in the low serum medium [13]. Briefly, hESCs were cultured on HFFs in 24 -well plates until reaching $80 \%$ confluency. They were then washed twice with PBS to remove serum in the SR medium. The medium was changed to RPMI supplemented with $2 \mathrm{mM} \mathrm{L}$-glutamine and $25 \mathrm{U} / \mathrm{mL}$ penicillin and $25 \mu \mathrm{g} / \mathrm{mL}$ streptomycin and varying concentrations of ESqualified FBS, $0 \%$ for the first day, $0.2 \%$ for the second day and $2 \%$ for subsequent days of differentiation. Activin A and/or Ex-4 (American Peptide Company, Sunnyvale, California, USA) were added to each experimental group for 5 days: group 1: Control; group 2: $100 \mathrm{ng} / \mathrm{mL}$ activin $\mathrm{A}$; group 3: $10 \mathrm{nM} \mathrm{Ex-4}$ and group 4: $100 \mathrm{ng} / \mathrm{mL}$ activin $\mathrm{A}+10$ $\mathrm{nM}$ Ex-4. Ex-4 concentrations used in this experiment was based on a previously published protocol [14]. The control group was hESCs cultured in the same medium in the absence of activin A or Ex-4. RNA samples were taken from undifferentiated hESCs (day 0) and from each condition on days 1,3 and 5 .

\section{Immunofluorescent Staining}

Prior to staining, hESCs were washed with PBS and fixed with $0.5 \mathrm{~mL}$ of $4 \%$ formaldehyde $/ 0.1 \%$ Triton $\mathrm{X}-100$ for $20 \mathrm{~min}$ at room temperature. After the cells were washed three times ( $3 \times 5 \mathrm{~min})$ with PBS, they were blocked with 0.5 $\mathrm{mL}$ of $1 \%$ FBS for 1 hour at room temperature. Primary and secondary antibodies were diluted in blocking solution. The cells were incubated with primary antibodies for overnight at $4^{\circ} \mathrm{C}$, washed 3 times in PBS and incubated with secondary antibodies for 1 hour at room temperature in dark. The following antibodies and dilutions were used: polyclonal rabbit anti-human GLP-1R (a gift from Dr. Daniel Drucker of the Banting and Best Diabetes Centre, University of Toronto), 1:1000; monoclonal mouse anti-human Sox17 (R\&D systems), 1:500; monoclonal goat anti-human Foxa2 (R\&D systems), 1:500; Alexa fluor 594 donkey anti-mouse IgG $(\mathrm{H}+\mathrm{L})$, 1:500, Alexa fluor 594 donkey anti-goat IgG $(\mathrm{H}+\mathrm{L}), 1: 500$; Alexa fluor 488 donkey anti-rabbit IgG $(\mathrm{H}+\mathrm{L})$, 1:500 (all secondary antibodies were from Molecular probes, Invitrogen Corporation). Negative staining controls were carried out by omitting primary antibodies. Cell nuclei were visualized by incubating with Prolong gold antifade reagent with DAPI (Molecular probes, Invitrogen Corporation). Images were captured using a Zeiss-Axioskop 2 microscope (Carl Zeiss Microimaging GmbH, Germany) using exposure times corresponding to negative controls. For quantification, positive cells were randomly counted against the total cell number (DAPI-positive cells). A minimum of 2,000 nuclei were counted in each staining.

\section{Characterization of hESCs by Quantitative Real Time Polymerase Chain Reaction (qPCR) Analysis}

Total RNA was prepared from RNeasy mini kits (Qiagen Pty Ltd). DNA contamination was removed by treating RNA samples with on-column RNase-free DNaseI (Qiagen Pty Ltd). cDNA was prepared by using $500 \mathrm{ng}$ of RNA. Standard reverse transcription was performed using SuperScript III First-Strand Synthesis System and Oligo (dT) primers (Invitrogen Corporation). Real-time PCR analysis was carried out on the Mx3500P Real-Time PCR system (Stratagene) using the SYBR Green PCR master mix from Integrated Sciences. The PCR reaction consisted of $10 \mu 1$ of SYBR Green PCR master Mix, $0.15 \mu 1$ of $10 \mu \mathrm{M}$ forward and reverse primers, $7.7 \mu$ l of nuclease-free water, and $2 \mu 1$ of diluted template cDNA in a total volume of $20 \mu 1$. Initial enzyme activation was performed at $95^{\circ} \mathrm{C}$ for $15 \mathrm{~min}$, followed by 40 cycles of denaturation at $95^{\circ} \mathrm{C}$ for $15 \mathrm{sec}$, and primer annealing/extension at $60^{\circ} \mathrm{C}$ for $1 \mathrm{~min}$. Melting curve analysis was performed at $95^{\circ} \mathrm{C}$ for $1 \mathrm{~min}, 60^{\circ} \mathrm{C}$ for $30 \mathrm{sec}$ and $95^{\circ} \mathrm{C}$ for $30 \mathrm{sec}$. The relative expression of each gene was normalized against the house-keeping gene, Beta-2 microglobulin (B2M). After normalization, each sample was plotted relative to undifferentiated hESCs. Each experiment was carried out at least 3 times.

\section{MicroRNA (miRNA) Analysis}

hESC differentiation to DE was carried out as previously described. hESCs were harvested using $1 \mathrm{mg} / \mathrm{mL}$ collagenase type IV (Sigma Aldrich, Saint Louis, Missouri, USA) and collected in TRIzol reagent (Invitrogen Corporation) (1 $\mathrm{mL}$ of TRIzol reagent/ 1 well of 24 -well plate) on day 0 (undifferentiated hESCs) and day 5 of differentiation (activin A-treated and activin A/Ex-4-treated samples) and stored at $80^{\circ} \mathrm{C}$. The miRNA analysis was performed following previously published protocols $[15,16]$. Total RNA was measured on ND-1000 spectrophotometer (NanoDrop Technologies, Wilminton, DE). Half of the RNA amount was used for reverse transcription using mature miRNA-specific primer sets (Applied Biosystems) while the remaining half was used for reverse transcription and qPCR using random primers (cDNA archival kit from Applied Biosystems). First strand cDNA synthesis was carried out using "high capacity cDNA transcription kit" (Applied Biosystems, Foster City, CA).

Complete human miRNA panel (Ver. 10.1, Applied Biosystems, Foster city, CA) including 283 miRNAs and 43 miRNAs from Early Access Kit (Applied Biosystems, Foster city, CA) was used. Reverse transcription was carried out using mature miRNA-specific primer sets (Applied Biosystems, Foster City, CA) and miRNA reverse transcription kit (Applied Biosystems, Foster City, CA). qPCR was performed on Applied Biosystems 7500 FAST system using miRNA-specific TaqMan-based probe-primer sets (Applied Biosystems, Foster City, CA). All sample plates including positive, negative and endogenous controls were supplied by the manufacturer in duplicate. Data are normalized to RNU44 miRNA levels and expressed as fold-changes relative to the undifferentiated hES3 cells. 


\section{Target Prediction and bi-Directional Cluster Analysis}

Since mammalian miRNAs are generally thought to recognize 3'UTR of target mRNA via partial complementtarity, carefully designed computational approaches were used to predict mRNA targets for miRNAs. Two target search engines including TargetScan Human prediction of miRNA targets, Release 5.1 developed by Whitehead Institute for biomedical research (http://www.targetscan.org/ vert_50/) and miRanda software target analysis by PicTar (http://pictar.mdc-berlin.de/cgi-bin/PicTar_vertebrate.cgi) were used to generate targets for specific miRNAs. The target gene lists were uploaded to Database for Annotation, Visualization and Integrated Discovery (DAVID) bioinformatics resources version 6.7 [17] and classified into Gene Ontology (GO) terms and Kyoto Encyclopedia of Genes and Genomes (KEGG) pathways. Furthermore, the functional analysis of highly regulated miRNAs was performed through the comparison with recently published reports.

Normalized data sets from qPCR analysis of miRNA expression profiles were taken as input data for bi-directional clustering. Two-way clustering was performed in MatLab ${ }^{\mathrm{TM}}$, using the Bioinformatics Tool-box (MatLab ${ }^{\mathrm{TM}}$ v 7.0, R 14), which groups samples with similar gene profiles together along the $\mathrm{X}$-axis. Genes with similar expression patterns are grouped along the $\mathrm{Y}$-axis.

The remaining half of RNA was used for TaqMan Low Density Array (TLDA) analysis. TLDA analysis was performed in $2 \mu \mathrm{L}$ reactions in 384-well TLDA plates using 40 ng cDNA input and TaqMan Fast Universal PCR Master Mix (Applied Biosystems, Foster City, CA). Primers and probes were Assay-on-Demand (Applied Biosystems). Data were normalized to GAPDH carried out using VIC-labeled probe in duplex reaction/well to correct for any differences in RNA input. Reactions were carried out on Applied Biosystems 7900 HT System.

\section{GLP-1R Signaling and Apoptosis}

Since the anti-apoptotic action of Ex-4 in hESCs is currently unknown, this experiment was designed to study the effect of Ex-4 in inhibiting apoptosis in hESCs cultured in a feeder-free system. hESCs were adapted to a defined and serum-free medium; mTeSR ${ }^{\mathrm{TM}} 1$ (StemCell Technologies, Vancouver, BC, Canada) [18] for at least one passage prior to experiments. In order to induce apoptosis, hESCs were cultured in KO-DMEM basal medium supplemented with 2 $\mathrm{mM}$ L-glutamine, $0.1 \mathrm{mM}$ non-essential amino acid, $1 \mathrm{X}$ insulin-transferrin-selenium, $25 \mathrm{U} / \mathrm{mL}$ penicillin, and 25 $\mu \mathrm{g} / \mathrm{mL}$ streptomycin. Withdrawal of bFGF and KnockOut ${ }^{\mathrm{TM}}$ serum replacement (SR) induces apoptosis and differenttiation [19]. In addition, withdrawal of 2-Mercaptoethanol from the culture medium also resulted in rapid inhibition of proliferation and subsequent cell death by apoptosis [20]. hESCs were cultured in mTeSR ${ }^{\mathrm{TM}} 1$ medium for 2 days to allow for cell attachment and proliferation. The following day, medium was changed to KO-DMEM medium and treated with bFGF and/or Ex-4 for 5 days as follows: Group 1: KODMEM; Group 2: KODMEM + $10 \mathrm{ng} / \mathrm{mL}$ bFGF; Group 3: KODMEM + $10 \mathrm{nM}$ Ex-4; Group 4: KODMEM + $100 \mathrm{nM}$ Ex-4; Group 5: KODMEM + $10 \mathrm{ng} / \mathrm{mL}$ bFGF + 10 nM Ex-4 and Group 6: mTeSR ${ }^{\mathrm{TM}} 1$ medium (control). RNA samples were extracted on days 2 and 5 . The expression levels of apoptotic markers, $C A S P 3, p 53$, and $p 21$, were examined by qPCR analysis.

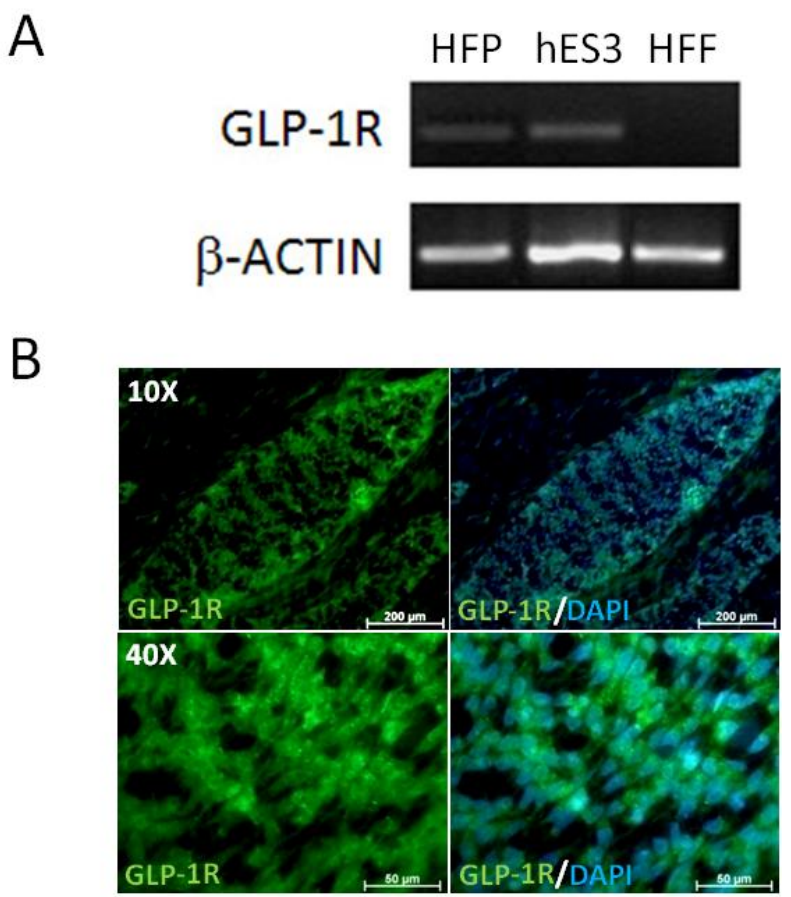

Fig. (1). GLP1-R expression in undifferentiated hESCs. (A) RT-PCR analysis for GLP-1R in hES3 cells, HFP: human fetal pancreas (positive control), HFF: human fetal fibroblasts (negative control). (B) Immunofluorescent staining for GLP-1R in hES3 cells. Scale bars $10 \mathrm{X}=200 \mu \mathrm{m}$ and $40 \mathrm{X}=50 \mu \mathrm{m}$. 


\section{Statistical Analyses}

The statistics generated in this study were performed using GraphPad Prism 5 (GraphPad Software, Inc). Significant difference was analyzed by one-way ANOVA analysis followed by Tukey-Kramer's multiple comparison tests. The results were considered significant $(*)$ when P-values were less than 0.05 .

\section{RESULTS}

\section{Determination of the GLP-1R Expression in Undiffe- rentiated hESCs}

Since GLP-1 and Ex-4 act through GLP-1R, it is essential to examine the presence of the GLP-1R on undifferentiated hESCs. We carried out RT-PCR as well as immunofluorescent staining of hESCs to determine the expression of the GLP-1R transcript and protein in hESCs. RT-PCR analysis confirmed the presence of the GLP-1R on undifferentiated hES3 cells (Fig. 1A). The GLP-1R staining in hES3 cells was localized within cytosol and plasma membrane as shown by immunofluorescent staining (Fig. 1B). HFF feeder layer (underneath the hESC colonies) did not express GLP-1R.

\section{The Effect of Ex-4 on hESCs and Activin A-Treated hESCs}

Undifferentiated hES3 colonies cultured in SR medium had a distinct boundary and the colonies were compact (Fig. 2A). Upon differentiation, these colonies became less compact and lost the defined boundary as observed on day 3 (Fig. 2B-2E). Ex-4-treated cells (Fig. 2D) had similar morphology as the untreated control cells (Fig. 2B) on day 3. However, activin A-treated cells (Fig. 2C) and the combined group (Fig. 2E) demonstrated similar changes in morphology consistent with complete differentiation.

\section{Analysis of Differentiated Markers}

Quantitative PCR (qPCR) analysis showed that exposure to $100 \mathrm{ng} / \mathrm{mL}$ activin $\mathrm{A}$ in the presence of low serum induced $\mathrm{SOX} 17$ and FOXA2 expressions from day 3 of
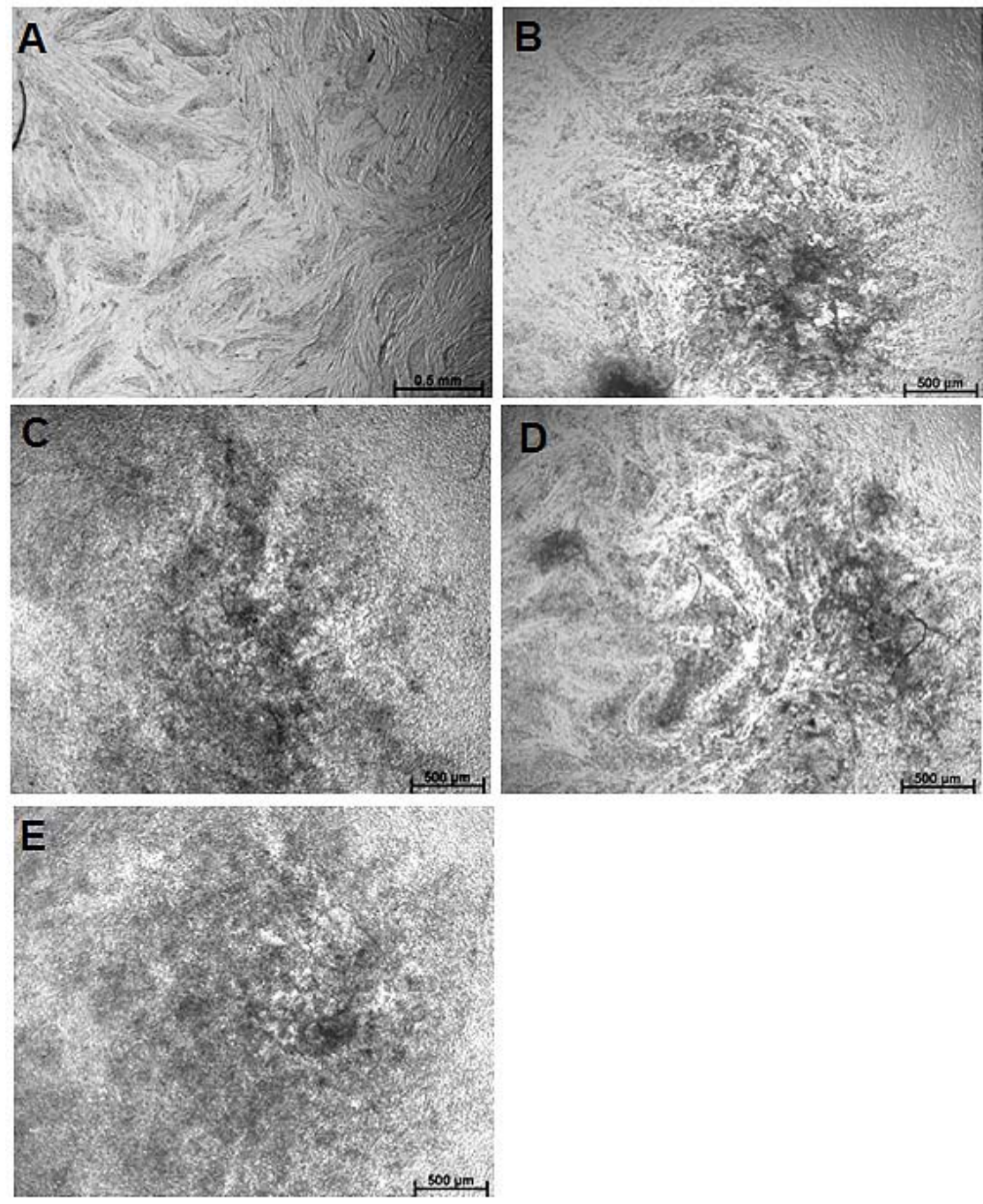

Fig. (2). Morphology of hESC colonies on day 0 and 3 days after differentiation. (A) Undifferentiated hES3 colonies on day 0 . Subsequent figures include: untreated control (B), activin A-treated hES3 cells (C), Ex-4-treated hES3 cells (D) and activin A/Ex-4-treated hES3 cells (E) after 3 days of differentiation. Scale bars $=500 \mu \mathrm{m}$. 
A

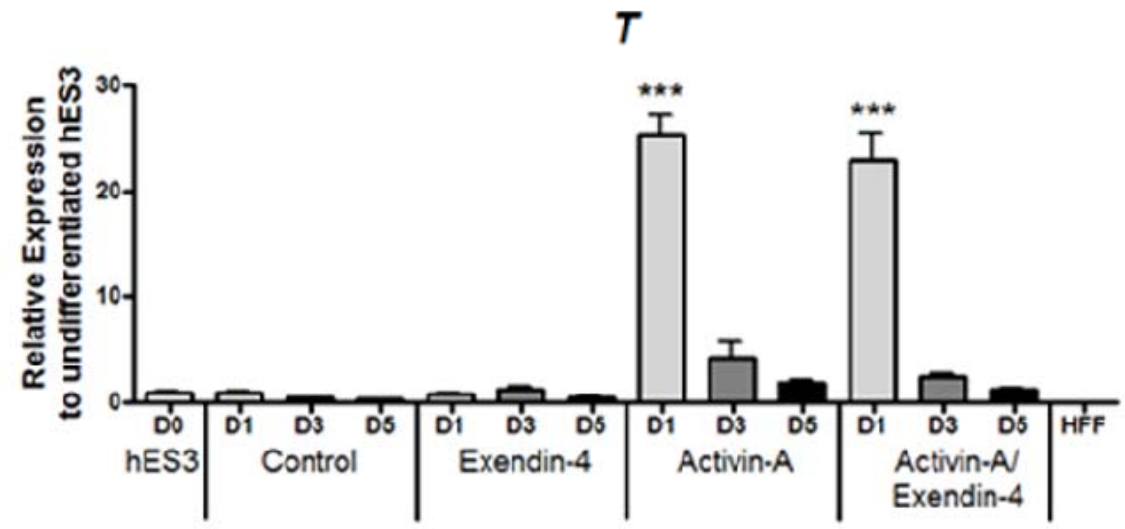

B

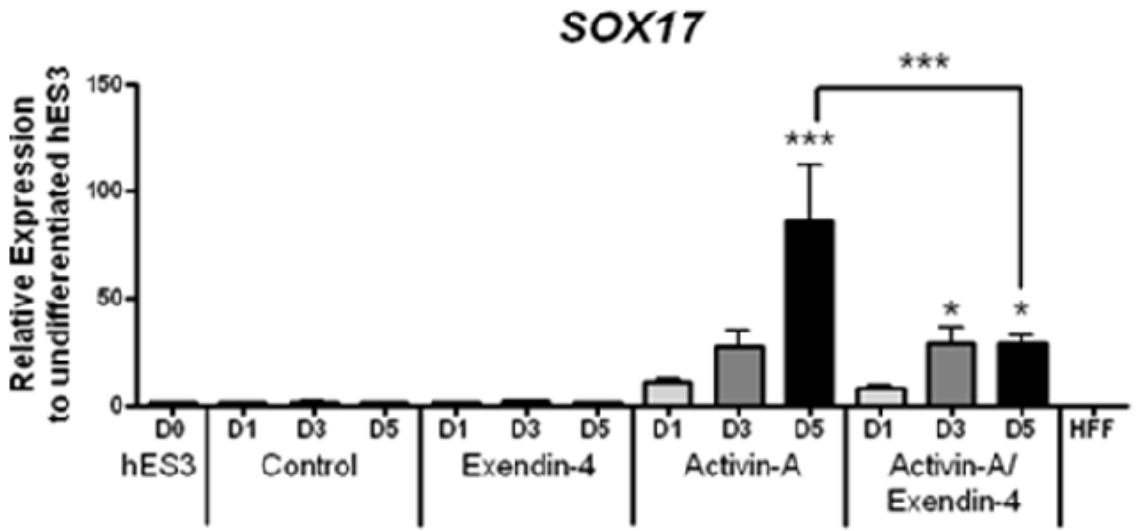

C

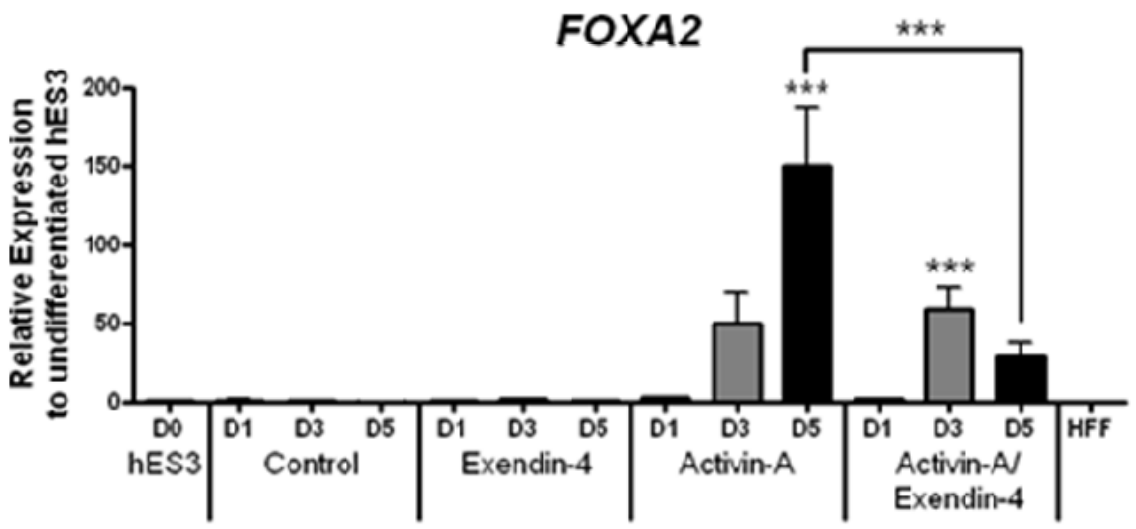

Fig. (3). qPCR analysis for the mesendodermal marker, brachyury (T) (A), and endodermal markers, SOX17 (B) and FOXA2 (C), on day 1,3 and 5 samples. The expression of each sample was normalized to the housekeeping gene, $B 2 M$. The fold change was compared relative to the undifferentiated hES3 cells (day 0). Data were presented as mean \pm S.D. with $\mathrm{N}=4$ experiments. $* \mathrm{P}<0.05, * * \mathrm{P}<0.01$ or $* * * \mathrm{P}<0.001$.

differentiation. The levels of SOX17 and FOXA2 were significantly upregulated on day 5 . Similarly, the combined group showed significant increases in SOX17 and FOXA2 expressions on day 3; however, these expression levels remained unchanged on day 5. Interestingly, the levels of both DE markers on day 5 were significantly higher in the activin A-treated group than the combined group. In contrast, the untreated control and Ex-4-treated cells did not express SOX17 and FOXA2 (Fig. 3B and 3C). To confirm if DE was from mesendodermal derived cells, we further carried out qPCR analysis of $T$ expression. Both activin A-treated and the combined group showed the peak of $T$ expression on day 1 followed by a substantial decrease on day 3 and 5 of differentiation. In contrast, little-to-no expression was observed in the untreated control and the Ex-4 treated cells (Fig. 3A). Protein expression was confirmed by immunofluorescent staining on day 5 of differentiation. There were more than $80 \%$ of SOX17-positive cells and FOXA2positive cells in activin A-treated samples and the combined groups. Similar levels of SOX17 and FOXA2 protein expressions were observed in the untreated control and Ex-4treated cells (Fig. 4A and 4B). The results from qPCR and immunofluorescent analyses confirmed that Ex-4 treatment alone had no significant effects on the expression of DE genes when compared to untreated control samples. In addition, the combination of activin $\mathrm{A}$ and Ex-4 treatment 


\section{A}
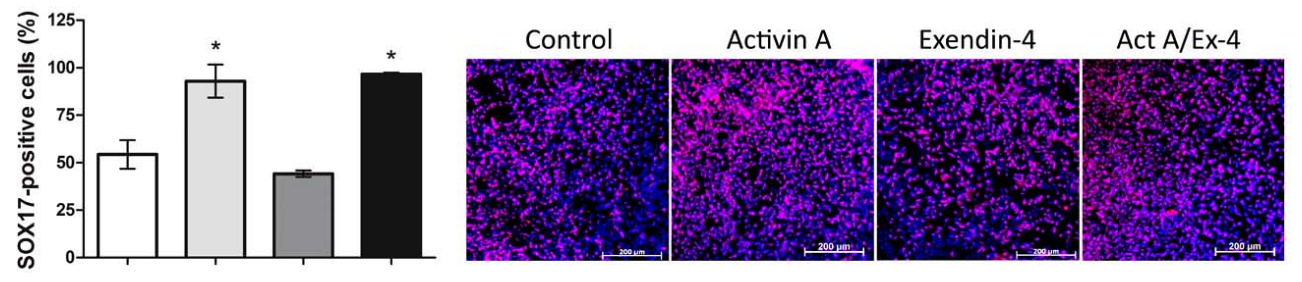

B
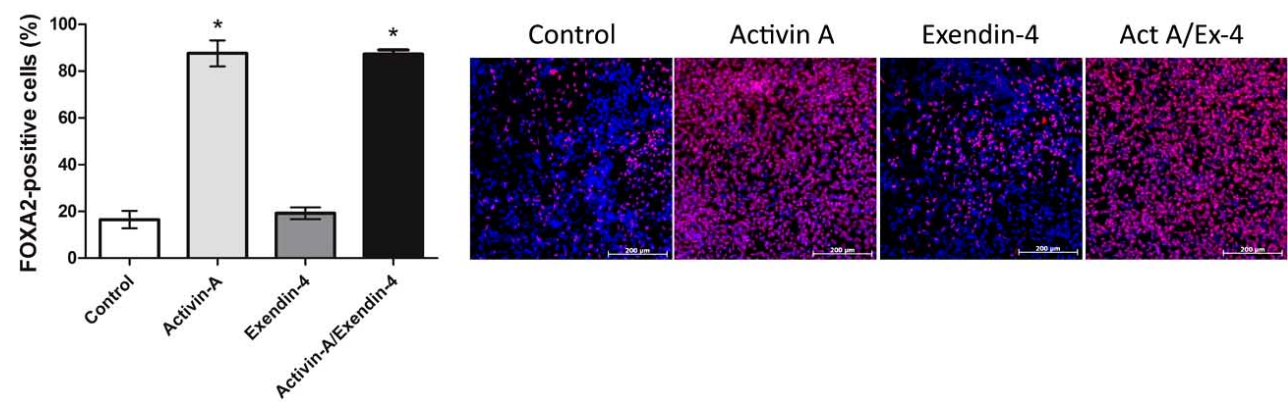

Fig. (4). Percentages of the SOX17 (A)- and FOXA2 (B)-positive cells on day 5 of differentiation. Positive cells were randomly counted against the total cell number (DAPI-positive cells). A total of at least 2,000 nuclei were counted in each staining. Data were presented as mean \pm S.D. with $\mathrm{N}=3$ experiments. $* \mathrm{P}<0.05$.

resulted in decreased expression of DE genes when compared to activin A-treated cells.

\section{Expression Profiling and Target Identification of miRNAs}

Several studies carried out until now have demonstrated that miRNAs can regulate gene expression by inducing RNA degradation or translational inhibition [21-25]. Since the expression levels of DE markers were significantly decreased $(\mathrm{P}<0.001)$ in the activin $\mathrm{A} / \mathrm{Ex}$-4-treated samples when compared with the activin A-treated samples (Fig. 3B and $3 \mathrm{C}$ ), the next set of experiments were designed to investigate miRNA expression profiles in both samples. The miRNA expression profiling of activin A-treated and activin A/Ex-4-treated hES3 cells as well as HFFs were quantified relative to undifferentiated hES3 cells. Changes in miRNA expression during hES3 differentiation to DE were presented

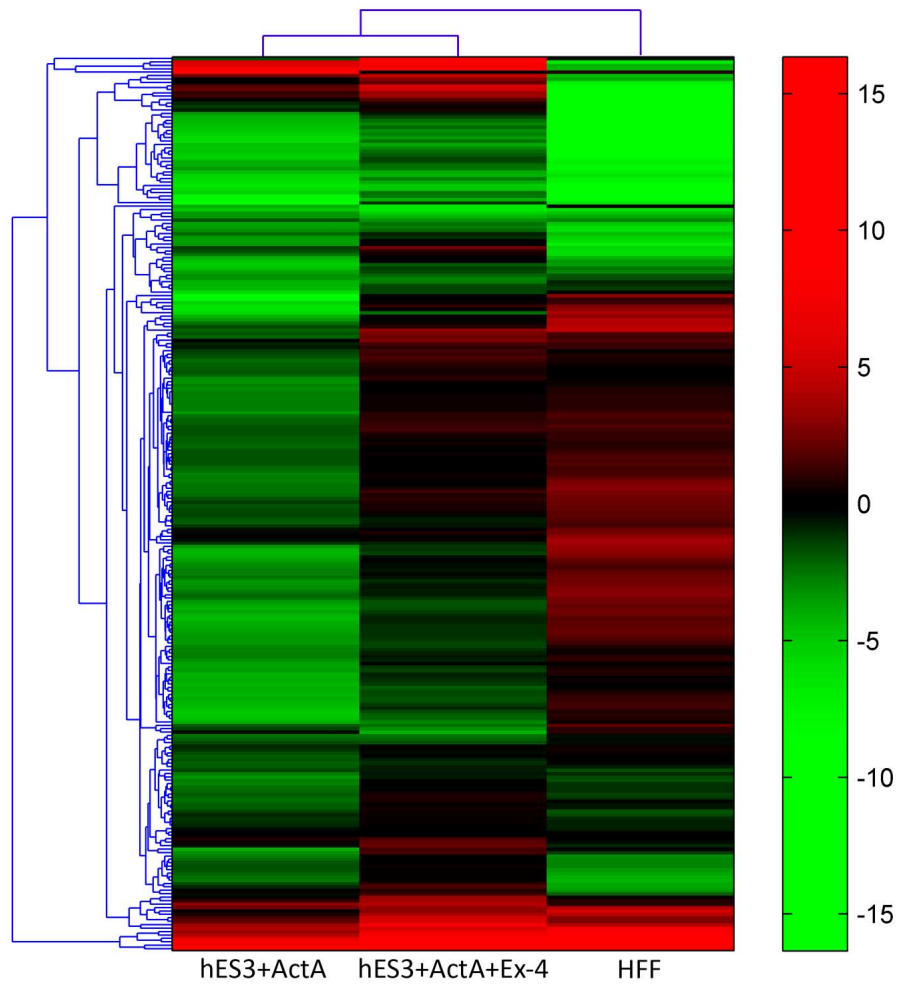

Fig. (5). Hierarchical clustering of miRNA expression of activin A-treated cells (hES3 + ActA), activin A/Ex-4-treated cells (hES3 + ActA + Ex-4) and human fetal fibroblasts (HFF). miRNAs are shown on the y-axis for each different cell sample on the x-axis. The scale bar represents the colour code for $\log _{2}$ fold-changes of different miRNAs as compared to undifferentiated hES3 cells as obtained by qPCR analysis ( $\mathrm{N}=3$ technical replicates from 1 biological repeat). 
Table 1. Representative miRNAs Differentially Expressed between Activin A-Treated Cells and Activin A/Ex-4-Treated Cells upon hESC Differentiation towards DE. List of top ten upregulated (A) and downregulated miRNAs (B) (fold changes of activin A/Ex-4-treated cells relative to activin A-treated cells with $N=3$ technical replicates). Of the 20 miRNAs, 3 were not registered in miRNA databases (red). Target genes of miRNAs were classified into GO terms (with the highest enrichment scores) and KEGG pathways (with the lowest corrected P-value)

A

\begin{tabular}{|c|c|c|c|}
\hline \multicolumn{2}{|c|}{ Upregulated miRNAs } & \multicolumn{2}{|c|}{ Target Gene Function } \\
\hline hsa-miR-423 & 271388.49 & N/A & N/A \\
\hline hsa-miR-213 & 968.76 & Regulation of kinase activity and phosphorylation & N/A \\
\hline hsa-miR-204 & 439.59 & Regulation of transcription, metal ion binding & Axon guidance \\
\hline hsa-miR-155 & 91.14 & Regulation of transcription & T-cell receptor signaling pathway \\
\hline hsa-miR-206 & 60.13 & Regulation of transcription & Neurotrophin signaling pathway \\
\hline hsa-miR-211 & 44.32 & Regulation of transcription and DNA binding & Axon guidance \\
\hline hsa-miR-520h & 39.67 & Cell adhesion and regulation of transcription & Axon guidance \\
\hline
\end{tabular}

B

\begin{tabular}{|c|c|c|c|}
\hline \multicolumn{2}{|c|}{ Downregulated miRNAs } & Target Gene Function \\
\hline miRNA ID & Fold Changes & Regulation of transcription and DNA binding & KEGG Pathway \\
\hline \hline hsa-miR-30e-3p & 0.000280444 & Protein kinase activity and nucleotide binding & Adipocytokine signaling pathway \\
\hline hsa-miR-33 & 0.080772052 & Regulation of transcription and DNA binding & Wnt signaling pathway \\
\hline hsa-miR-381 & 0.164938489 & Regulation of translation and RNA binding & Chronic myeloid leukemia \\
\hline hsa-miR-526b & 0.301451957 & Metal ion binding and regulation of transcription & N/A \\
\hline hsa-miR-496 & 0.316439148 & N/A & N/A \\
\hline hsa-miR-513 & 0.320856474 & RNA and nucleotide binding & N/A \\
\hline hsa-miR-521 & 0.343885455 & Protein kinase activity and nucleotide binding & mTOR signaling pathway \\
\hline hsa-miR-122a & 0.539614118 & Regulation of phosphorylation/kinase activity & N/A \\
\hline hsa-miR-451 & 0.678302164 & N/A & \\
\hline hsa-miR-450 & 0.768437591 & & \\
\hline
\end{tabular}

as a dendrogram, which indicates that majority of miRNAs in the activin A-treated cells were downregulated when compared with the undifferentiated cells and the activin A/Ex-4treated cells (Fig. 5). Examination of the miRNA expression profiles revealed miRNAs that were differentially expressed between the two treatments. Table 1 demonstrates the top ten upregulated and downregulated miRNAs (presented as fold changes), which were differentially expressed in activin A/Ex-4-treated cells relative to activin A-treated cells. Of the 20 miRNAs, 3 were not registered and were excluded from the analysis (Table 1, red). Using DAVID bioinformatics resources [17], the target gene list of each miRNA was categorised into GO terms and KEGG pathways as demonstrated in Table 1. To assess the effect of Ex-4 treatment during hES3 differentiation, miRNA expression profiles were grouped in terms of pluripotency (Supplemen- tary Fig. S1) and endoderm/pancreas-associated (Supplementary Fig. S2) miRNAs according to previous studies [15, 26-33]. Pluripotency-associated miRNAs were presented in Fig. (6). qPCR analysis indicated that miR-302a* and miR$302 c^{*}$ were expressed at high levels in activin A/Ex-4treated hES3 cells (hES3 + ActA + Ex-4). These two miRNAs are members of the miR-302 family (miR-302s), which are expressed abundantly in hESCs [33] and have been reported to maintain hESC pluripotency and self renewal [34]. Expression of endoderm-specific miRNAs (miR-24, miR-10a), FOXA2 suppressor (miR-124a), HES1 suppressor (miR-23a, miR-23b), pancreas-specific miRNAs (miR-214, miR-7, miR-9) and islet-specific miRNA (miR375, miR-376a) were comparable in both activin A-treated and activin A/Ex-4-treated hES3 cells (Supplementary Fig. S2). 


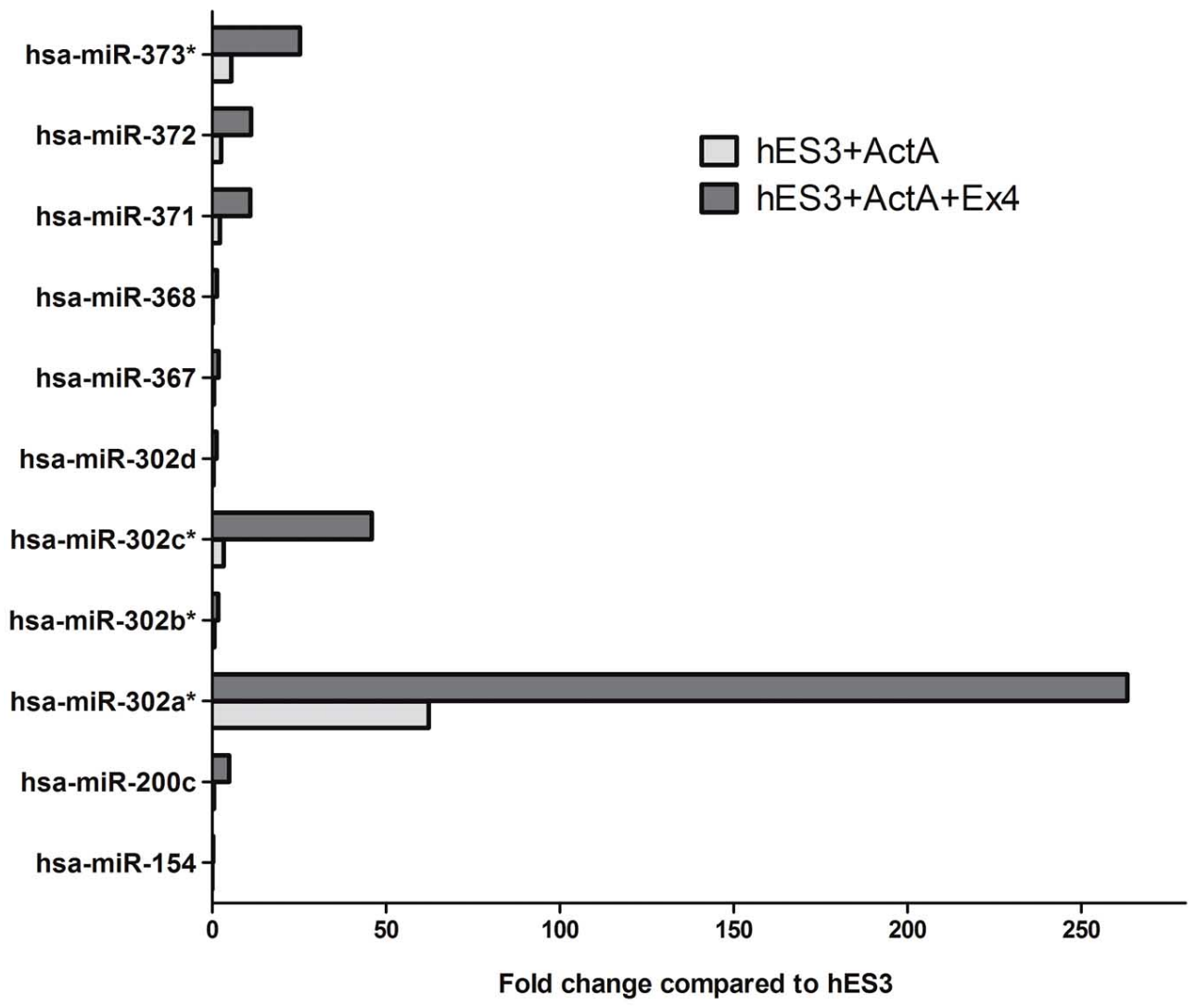

Fig. (6). Pluripotency-associated miRNA expression of activin A-treated cells (hES3 + ActA) and activin A/Ex-4-treated cells (hES3 + ActA + Ex-4). Data were expressed as fold changes relative to undifferentiated hES3 cells $(\mathrm{N}=3$ technical replicates).

RNA samples were used for reverse transcription and qPCR analysis. Hierarchical clustering of gene expression profiles during DE differentiation with and without Ex-4 supplementation was demonstrated in Table 2. Six out of ten

Table 2. Upregulated Genes upon hES3 Differentiation to DE. List of the ten mRNA transcripts showing the largest increase (fold changes) upon activin $A$ and/or Ex-4 treatments for 5 days. Expression is presented as fold changes relative to undifferentiated hES3 cells (day 0) $(\mathbf{N}=\mathbf{3}$ technical replicates). The genes expressed in both samples are shown in blue

\begin{tabular}{|c|c|c|c|}
\hline \multicolumn{2}{|c|}{ hES3 + ActA } & \multicolumn{2}{c|}{ hES3 + ActA + Ex-4 } \\
\hline Gene & Fold Changes & Gene & Fold Changes \\
\hline \hline GATA4 & 1563506.73 & GATA4 & 1363067.90 \\
\hline PAX6 & 256.39 & HLXB9 & 67.93 \\
\hline HLXB9 & 72.69 & CXCR4 & 58.81 \\
\hline CXCR4 & 48.16 & GATA6 & 41.23 \\
\hline GATA6 & 44.72 & NCAM1 & 4.12 \\
\hline PAX4 & 6.68 & FOXJ2 & 3.85 \\
\hline NCAM1 & 3.78 & PAX4 & 3.06 \\
\hline GCK & 3.74 & GAL & 2.92 \\
\hline FOXJ2 & 3.02 & P4HA1 & 2.91 \\
\hline P4HA1 & 2.95 & GCK & 2.80 \\
\hline
\end{tabular}

genes, which exhibited the greatest upregulation upon treatments with activin A and/or Ex-4, were endoderm and pancreas-related genes: GATA4, GATA6, CXCR4, HLXB9, $P A X 4$, and $G C K$. The expressions of endoderm and pancreas-related genes were comparable in both samples. These data indicated that the cells from both treatments differrentiated towards endoderm and pancreatic cells.

\section{GLP-1R Signaling and Apoptosis}

It was demonstrated that GLP-1R is expressed in undifferentiated hESCs; however, the role of Ex-4 on hESCs is currently unknown. Firstly, it was postulated that GLP-1R may be activated by Ex-4 and thus potentiates DE differrentiation. However, it was demonstrated that Ex-4 significantly inhibited the expression levels of SOX17 and FOXA2 transcripts during hESC differentiation to DE (Fig. 3B and 3C). Furthermore, the data from miRNA study demonstrates that pancreas-associated miRNAs were expressed at similar levels in both samples. Since Ex-4 had no role in enhancing DE differentiation under these conditions, we postulated that it may have other role(s) in undifferentiated hESCs. We therefore examined if Ex-4 prevented apoptosis in pluripotent hESCs as observed in other fully differentiated cells including freshly isolated islets [4] and hippocampal and DA neurons [9, 11, 35]. hESCs were cultured in $\mathrm{mTeSR}^{\mathrm{TM}} 1$ medium on Matrigelcoated surfaces for the first two days. The following day, medium was changed to KO-DMEM medium, which did not contain $\beta$-mercaptoethanol, bFGF and SR, therefore, this medium would inhibit proliferation and induce apoptotic cell death $[19,20]$. hESCs were then supplemented with bFGF 


\section{Caspase-3}
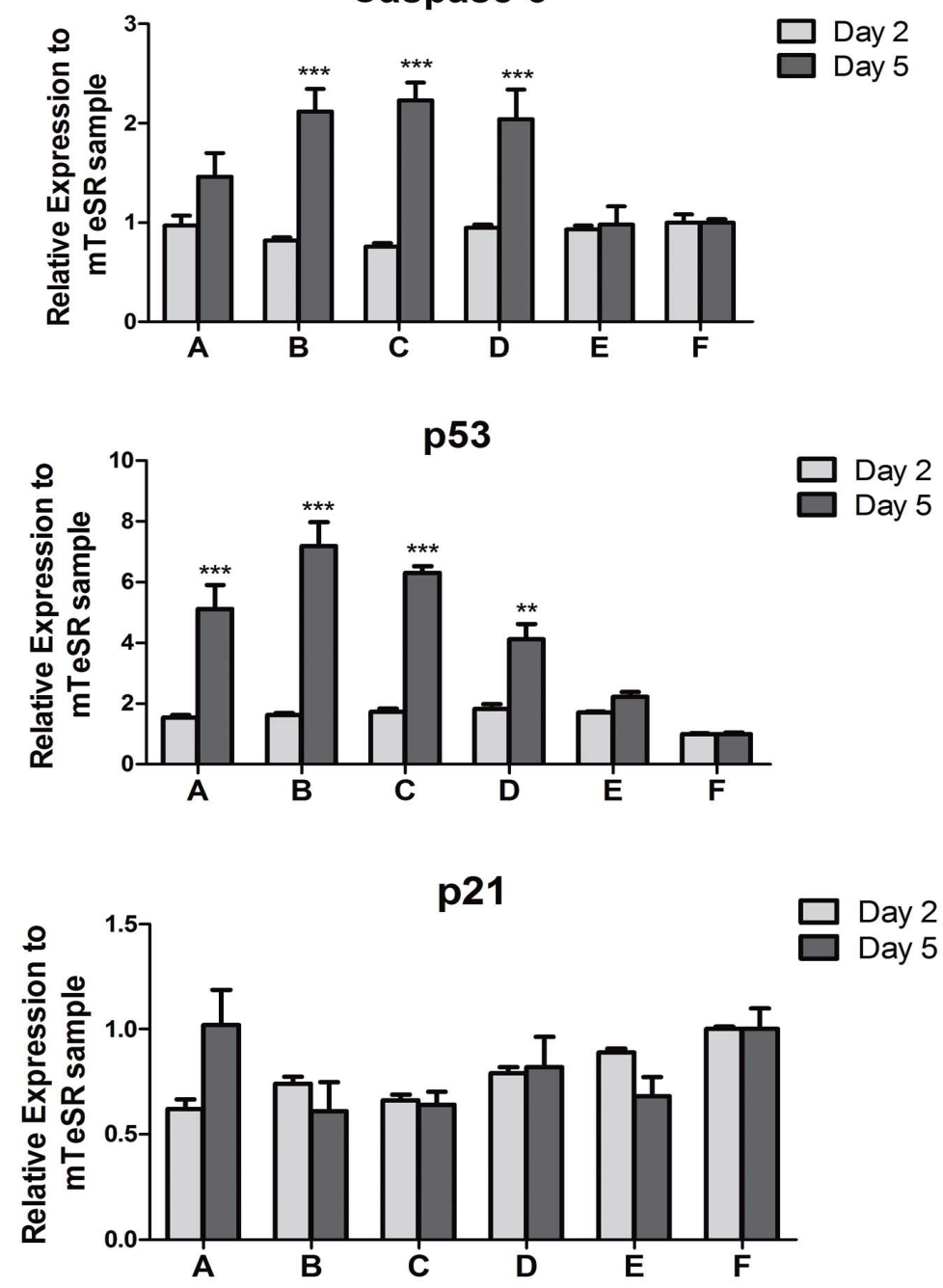
A. KO-DMEM basal medium
B. KO-DMEM + $10 \mathrm{ng} / \mathrm{mL}$ bFGF
C. KO-DMEM + 10 nM Ex-4
D. KO-DMEM + $100 \mathrm{nM}$ Ex-4
E. $K O-D M E M+10 \mathrm{ng} / \mathrm{mL}$ bFGF + $10 \mathrm{nM}$ Ex-4
F. mTESR $^{\mathrm{TM}}$ medium

Fig. (7). qPCR analysis for apoptotic markers following treatments with Ex-4 and/or bFGF on pluripotent hESCs under feeder-free condition. The expression of each sample was normalized with housekeeping gene, B2M. The fold changes were compared relative to hESCs cultured in $\mathrm{mTeSR}^{\mathrm{TM}} 1$ medium $(\mathrm{F})$ on respective day. Data presented as mean \pm S.D. $(\mathrm{N}=3)$. The results were considered significant when $* \mathrm{P}<0.05, * * \mathrm{P}<0.01$ or $* * * \mathrm{P}<0.001$.

and/or Ex-4 for the next 5 days. Cell detachment was observed under all conditions indicating that the cells may be undergoing apoptosis. qPCR analysis of apoptotic markers was then performed on day 2 and day 5 after the treatment. The levels of apoptotic markers, CASP3 and p53, were significantly increased in day 5 samples as compared to day 2 samples in the following conditions: KO-DMEM medium (A), KO-DMEM medium + bFGF (B), KO-DMEM medium + 10 nM Ex-4 (C), KO-DMEM medium + 100 nM Ex-4 (D). However, Ex-4 treatment in combination with bFGF treatment (KO-DMEM medium + bFGF + Ex-4) (E) reduced the levels CASP3 and p53 in day 5 samples, to similar levels of cells cultured in $\mathrm{mTeSR}^{\mathrm{TM}} 1$ medium $(\mathrm{F})$. The expression levels of the apoptotic marker $p 21$, which is downstream of p53 [36], were not significantly different in all samples (Fig. 7).

\section{DISCUSSION}

Recent studies in hESCs have shown that they can be directed to differentiate to DE [13]. This is the first step towards creating insulin-producing cells. GLP-1 plays important roles in stimulating $\beta$-cell formation and insulin secretion as well as preventing $\beta$-cell apoptosis $[1,4]$. Several reports demonstrated that GLP-1 and Ex-4 stimulate differentiation of stem/progenitor cells to an endocrine 
phenotype and induce the expression of insulin, glucagon and other islet endocrine cell markers [37-39]. The role of Ex-4 on hESC endodermal differentiation is currently unknown. The present study aimed at examining the effects of Ex-4 alone and in combination with activin A on hESCs viability and differentiation. Firstly, it was essential that the GLP-1R is present in hESCs since its N-terminal extracellular region is required for GLP-1 or Ex-4 binding [40]. The effects of Ex-4 on hESCs and the combined effects of Ex-4 and activin A-treated cells were examined using a 5day protocol to derive DE [13]. Ex-4 supplementation (i.e. activin A/Ex-4 treated cells) did not affect $T$ expression levels; however, the combination of activin A and Ex-4 resulted in a significant decrease in DE transcript levels. However, the decrease in DE expression was not observed at the protein levels. Ex-4 treatment alone had no significant effects on the expression of mesendodermal and DE genes.

In contrast to the present study, GLP-1 was previously used to enhance the production of insulin in mESC-derived insulin-producing cells at the later stages of differentiation [14] by modifying a previously described five-stage protocol [41]. GLP-1 (or Ex-4) was supplemented to the differentiation medium during the last stage (stage 5) to obtain insulin-producing cells. Their study also demonstrated that GLP-1R transcript and protein were detected at all stages of differentiation [14]. It was also suggested that the GLP-1R in undifferentiated mESCs may serve no function as ESCs have been demonstrated to express many tissue-specific genes with no apparent roles [42]. Furthermore, hESCs were differrentiated using the five-stage protocol with Ex-4 supplementation in stage 4 and 5 to generate insulin-producing cells [43]. Together, these studies suggested that Ex-4 in combination with other growth factors may aid in the generation of insulin-producing cells at the later stages of differentiation. It is possible that the cells at the later stages (stage 4 or 5 ) derived from these protocols are similar to endocrine pancreatic progenitors, which can be induced by Ex-4 and other growth factors to produce islet hormones [37$39,44]$.

It was believed that Ex-4 may have a subtle but important regulatory effect on gene expression during DE differenttiation. Therefore, further investigation was performed to examine miRNA expression profiles of activin A-treated and activin A/Ex-4-treated cells. Recent studies have demonstrated that miRNAs are important regulators of posttranscriptional gene expression by causing RNA degradation hence inhibiting translation [45]. The present study compared miRNA expression profiles between activin A-treated hESCs and activin A/Ex-4-treated hESCs after 5 days of treatment. Endoderm-specific miRNAs (miR-24, miR-10a), FOXA2 suppressor (miR-124a), HES1 suppressor (miR-23a, miR-23b), pancreas-specific miRNAs (miR-214, miR-7) and islet-specific miRNAs (miR-375, miR-376a) were expressed at similar levels in both samples. The expression of miR-375 observed in the present study was consistent with a previous study which demonstrated that miR-375 was not only expressed during pancreatic development but also highly upregulated during endodermal differentiation [46].

Analysis of endoderm and pancreas-related mRNA expression levels such as GATA4, GATA6, CXCR4, HLXB9, $P A X 4$, and $G C K$ demonstrated upregulation upon differen- tiation in both activin A-treated and activin A/Ex-4-treated hESCs. However, our data also demonstrated that the expression levels of two DE markers, SOXI7 and FOXA2, were significantly downregulated in activin A/Ex-4-treated hESCs. Due to the highly upregulated expression levels of pluripotency-associated miRNAs, miR-302a* and miR$302 \mathrm{c}^{*}$ [26], in activin $\mathrm{A} / \mathrm{Ex}-4$-treated $\mathrm{hESCs}$, it was possible that addition of Ex-4 resulted in this upregulation, which could then lead to inhibition of SOXI7 and FOXA2 expression. However, functional analysis involving overexpression /knockdown studies for specific miRNAs using stable expression systems need to be performed.

Previous studies have shown that GLP-1R is widely detected in many tissues including endocrine pancreas, intestinal tract, brain, lung, kidney, heart [8] and mouse skin [47]. In cells harbouring GLP-1R, it was demonstrated that GLP-1 promotes direct resistance to apoptosis through Gprotein coupled receptor interaction leading to activation of adenylyl cyclase (increase in cAMP), protein kinase $\mathrm{C}$ and mitogen activated protein in diverse cell types $[48,49]$. The anti-apoptotic properties of GLP-1 agonists have been demonstrated in diabetic rodents [5], freshly isolated human islets [4], and fetal rat hippocampal and cholinergic neurons [50]. GLP-1 also increased cell survival and reduced caspase activation in baby hamster kidney (BHK) fibroblasts expressing a transfected GLP-1R [5]. As a result, these studies suggest that direct coupling to anti-apoptotic signaling pathways may represent a generalized role of GLP-1 and its receptor in various cell types. Therefore, the subsequent study was also designed to study the effect of Ex-4 on hESCs cultured in apoptotis-inducing medium. Treatment with bFGF or Ex-4 alone was not sufficient to reduce the levels of $C A S P 3$ and $p 53$. However, in the samples cultured in the presence of both bFGF and Ex-4, the levels of both apoptotic markers significantly decreased to levels similar to those cells cultured in $\mathrm{mTeSR}^{\mathrm{TM}} 1$ medium. This result was consistent with a previous study, which demonstrated that bFGF prevents ESCs from apoptosis via inhibition of caspase activation [19] and suggested that bFGF may work in synergy with Ex-4 to protect hESCs from apoptosis.

\section{CONCLUSION}

We demonstrate here the presence of GLP-1R on hESCs using RT-PCR and immunofluorescent analysis. However, Ex-4 mediated signaling via GLP-1R did not enhance DE formation in hESCs. On the other hand, a combinatorial treatment with activin A and Ex-4 resulted in a significant decrease in the expression levels of DE markers. The miRNA expression profiles between activin A-treated hESCs and activin A/Ex-4-treated hESCs after 5 days of treatment demonstrated similar expression levels of endoderm and pancreas-associated miRNAs. However, it was observed that levels of pluripotency-associated miRNAs, miR-302a* and miR302 $c^{*}$, were upregulated in the presence of Ex-4. Furthermore, it was demonstrated that the combination of bFGF and Ex-4 treatment in apoptosis-inducing medium resulted in downregulation of $C A S P 3$ and $p 53$. While these data reveal the possible role of Ex-4 in maintaining pluripotency and inhibiting apoptosis, the knowledge of GLP-1 signaling pathways in hESCs is still lacking. A full 
understanding of mechanism of GLP-1 and its receptor will require future experimentations.

\section{ACKNOWLEDGEMENTS}

We thank Dr. Bernard E. Tuch for providing laboratory space and facilitating this study. We also thank Dr. Mugdha V. Joglekar from Stem Cells and Diabetes Section, National Center for Cell Science, India for her assistance with miRNA analysis. This study was supported by Stem Cell initiative from the Faculty of Medicine, The University of New South Wales.

\section{ABBREVIATIONS}

hESCs = Human embryonic stem cells

miRNAs $=$ microRNAs

GLP-1 = Glucagon-like peptide-1

Ex-4 = Exendin-4

\section{SUPPLEMENTARY MATERIALS}

This article is also accompanied with supplementary material and it can be viewed at publisher's web site.

\section{REFERENCES}

[1] Drucker DJ. Glucagon-like peptides: regulators of cell proliferation, differentiation, and apoptosis. Mol Endocrinol 2003; 17(2): 161-71.

[2] Xu G, Kaneto H, Lopez-Avalos MD, Weir GC, Bonner-Weir S. GLP-1/exendin-4 facilitates b-cell neogenesis in rat and human pancreatic ducts. Diabetes Res Clin Pract 2006; 73(1): 107-10.

[3] Tourrel C, Bailbe D, Meile M-J, Kergoat M, Portha B. GlucagonLike Peptide-1 and Exendin-4 Stimulate $\beta$-Cell neogenesis in streptozotocin-treated newborn rats resulting in persistently improved glucose homeostasis at adult age. Diabetes 2001; 50(7): 1562-70.

[4] Farilla L, Bulotta A, Hirshberg B, et al. Glucagon-like peptide 1 inhibits cell apoptosis and improves glucose responsiveness of freshly isolated human islets. Endocrinology 2003; 144(12): 514958.

[5] Li Y, Hansotia T, Yusta B, Ris F, Halban PA, Drueker DJ. Glucagon-like peptide-1 receptor signaling modulates $\beta$ cell apoptosis. J Biol Chem 2003; 278(1): 471-8.

[6] Goke R, Fehmann HC, Linn T, et al. Exendin-4 is a high potency agonist and truncated exendin-(9-39)-amide an antagonist at the glucagon-like peptide 1-(7-36)-amide receptor of insulin- secreting $\hat{\mathrm{I}}^{2}$-cells. J Biol Chem 1993; 268(26): 19650-5.

[7] Monami M, Marchionni N, Mannucci E. Glucagon-like peptide-1 receptor agonists in type 2 diabetes: a meta-analysis of randomized clinical trials. Eur J Endocrinol 2009; 160(6): 909-17.

[8] Wei Y, Mojsov S. Tissue-specific expression of the human receptor for glucagon-like peptide-I: brain, heart and pancreatic forms have the same deduced amino acid sequences. FEBS Lett 1995; 358(3): 219-24.

[9] Perry T, Lahiri DK, Sambamurti K, et al. Glucagon-like peptide-1 decreases endogenous amyloid-beta peptide (Abeta) levels and protects hippocampal neurons from death induced by Abeta and iron. J Neurosci Res 2003; 72(5): 603-12.

[10] Qin Z, Sun Z, Huang J, Hu Y, Wu Z, Mei B. Mutated recombinant human glucagon-like peptide-1 protects SH-SY5Y cells from apoptosis induced by amyloid- $\hat{\mathrm{I}}^{2}$ peptide (1-42). Neurosci Lett 2008; 444(3): 217-21.

[11] Harkavyi A, Abuirmeileh A, Lever R, Kingsbury AE, Biggs CS, Whitton PS. Glucagon-like peptide 1 receptor stimulation reverses key deficits in distinct rodent models of Parkinson's disease. J Neuroinflammation 2008; 5: 19.
[12] Sidhu KS, Ryan JP, Tuch BE. Derivation of a new human embryonic stem cell line, endeavour-1, and its clonal propagation. Stem Cells Dev 2008; 17(1): 41-51.

[13] D'Amour KA, Agulnick AD, Eliazer S, Kelly OG, Kroon E, Baetge EE. Efficient differentiation of human embryonic stem cells to definitive endoderm. Nat Biotechnol 2005; 23(12): 1534-41.

[14] Bai L, Meredith G, Tuch BE. Glucagon-like peptide-1 enhances production of insulin in insulin-producing cells derived from mouse embryonic stem cells. J Endocrinol 2005; 186(2): 343-52.

[15] Joglekar MV, Joglekar VM, Hardikar AA. Expression of isletspecific microRNAs during human pancreatic development. Gene Expr Patterns 2009; 9(2): 109-13.

[16] Joglekar MV, Parekh VS, Mehta S, Bhonde RR, Hardikar AA. MicroRNA profiling of developing and regenerating pancreas reveal post-transcriptional regulation of neurogenin3. Dev Biol 2007; 311(2): 603-12.

[17] Huang DW, Sherman BT, Lempicki RA. Systematic and integrative analysis of large gene lists using DAVID bioinformatics resources. Nat Protoc 2009; 4(1): 44-57.

[18] Ludwig TE, Levenstein ME, Jones JM, et al. Derivation of human embryonic stem cells in defined conditions. Nat Biotechnol 2006; 24(2): 185-7.

[19] Wang X, Lin G, Martins-Taylor K, Zeng H, Xu R-H. Inhibition of caspase-mediated anoikis is critical for bFGF-sustained culture of human pluripotent stem cells. J Biol Chem 2009; 284: 34054-64.

[20] Neumann D, Zierke M, Martin MU. Withdrawal of 2mercaptoethanol induces apoptosis in a B-cell line via fas upregulation. J Cell Physiol 1998; 177(1): 68-75.

[21] Jackson RJ, Standart N. How do microRNAs regulate gene expression? Sci STKE 2007; 2007(367): P. re1.

[22] Schmitter D, Filkowski J, Sewer A, et al. Effects of dicer and argonaute down-regulation on mRNA levels in human HEK293 cells. Nucleic Acids Res 2006; 34(17): 4801-15.

[23] Behm-Ansmant I, Rehwinkel J, Doerks T, Stark A, Bork P, Izaurralde E. mRNA degradation by miRNAs and GW182 requires both CCR4:NOT deadenylase and DCP1:DCP2 decapping complexes. Genes Dev 2006; 20(14): 1885-98.

[24] Wu L, Fan J, Belasco JG. MicroRNAs direct rapid deadenylation of mRNA. Proc Natl Acad Sci USA 2006; 103(11): 4034-9.

[25] Joglekar MV, Parekh VS, Hardikar AA. New pancreas from old: microregulators of pancreas regeneration. Trends Endocrinol Metab 2007; 18(10): 393-400.

[26] Barroso-Del Jesus A, Lucena-Aguilar G, Menendez P. The miR302-367 cluster as a potential stemness regulator in ESCs. Cell Cycle 2009; 8(3): 394-8.

[27] Barroso-Del Jesus A, Romero-L $\tilde{A}^{3}$ pez C, Lucena-Aguilar G, et al. Embryonic stem cell-specific miR302-367 cluster: Human gene structure and functional characterization of its core promoter. Mol Cell Biol 2008; 28(21): 6609-19.

[28] Chivukula RR, Mendell JT. Abate and Switch: miR-145 in Stem Cell Differentiation. Cell 2009; 137(4): 606-8.

[29] Gangaraju VK, Lin H. MicroRNAs: Key regulators of stem cells. Nat Rev Mol Cell Biol 2009; 10(2): 116-25.

[30] Poy MN, Eliasson L, Krutzfeldt J, et al. A pancreatic islet-specific microRNA regulates insulin secretion. Nature 2004; 432(7014): 226-30.

[31] Ren J, Jin P, Wang E, Marincola FM, Stroncek DF. MicroRNA and gene expression patterns in the differentiation of human embryonic stem cells. J Transl Med 2009; 7: 20.

[32] Tzur G, Levy A, Meiri E, et al. MicroRNA expression patterns and function in endodermal differentiation of human embryonic stem cells. PLoS One 2008; 3(11): e3726.

[33] Suh MR, Lee Y, Kim JY, et al. Human embryonic stem cells express a unique set of microRNAs. Dev Biol 2004; 270(2): 48898.

[34] Lin SL, Chang DC, Chang-Lin S, et al. Mir-302 reprograms human skin cancer cells into a pluripotent ES-cell-like state. RNA 2008; 14(10): 2115-24

[35] Qin Z, Sun Z, Huang J, Hu Y, Wu Z, Mei B. Mutated recombinant human glucagon-like peptide-1 protects SH-SY5Y cells from apoptosis induced by amyloid- $\beta$ peptide (1-42). Neurosci Lett 2008; 444(3): 217-21.

[36] Gartel AL, Serfas MS, Tyner AL. p21 - Negative regulator of the cell cycle. Proc Soc Exp Biol Med 1996; 213(2): 138-49.

[37] Hui H, Wright C, Perfetti R. Glucagon-like peptide 1 induces differentiation of islet duodenal homeobox-1-positive pancreatic 
ductal cells into insulin-secreting cells. Diabetes 2001; 50(4): 78596.

[38] Zhou J, Pineyro MA, Wang X, Doyle ME, Egan JM. Exendin-4 differentiation of a human pancreatic duct cell line into endocrine cells: Involvement of PDX-1 and HNF3beta transcription factors. J Cell Physiol 2002; 192(3): 304-14.

[39] Zhou J, Wang X, Pineyro MA, Egan JM. Glucagon-like peptide 1 and exendin-4 convert pancreatic AR42J cells into glucagon- and insulin-producing cells. Diabetes 1999; 48(12): 2358-66.

[40] Baggio LL, Drucker DJ. Biology of incretins: GLP-1 and GIP. Gastroenterology 2007; 132(6): 2131-57.

[41] Lumelsky N, Blondel O, Laeng P, Velasco I, Ravin R, McKay R. Differentiation of embryonic stem cells to insulin-secreting structures similar to pancreatic islets. Science 2001; 292(5520): 1389-94.

[42] Friedrich G, Soriano P. Promoter traps in embryonic stem cells: a genetic screen to identify and mutate developmental genes in mice. Genes Dev 1991; 5(9): 1513-23.

[43] D'Amour KA, Bang AG, Eliazer S, et al. Production of pancreatic hormone-expressing endocrine cells from human embryonic stem cells. Nat Biotechnol 2006; 24(11): 1392-401.
[44] Hardikar AA, Wang XY, Williams LJ, et al. Functional maturation of fetal porcine beta-cells by glucagon-like peptide 1 and cholecystokinin. Endocrinology 2002; 143(9): 3505-14.

[45] Bushati N, Cohen SM. MicroRNA functions. Ann Rev Cell Dev Biol 2007; 23: 175-205.

[46] Hinton A, Afrikanova I, Wilson M, et al. A Distinct MicroRNA signature for definitive endoderm derived from human embryonic stem cells. Stem Cells Dev 2009; 19(6): 797-807.

[47] List JF, He H, Habener JF. Glucagon-like peptide-1 receptor and proglucagon expression in mouse skin. Regul Pept 2006; 134(2-3): $149-57$.

[48] Montrose-Rafizadeh C, Avdonin P, Garant MJ, et al. Pancreatic glucagon-like peptide-1 receptor couples to multiple $\mathrm{G}$ proteins and activates mitogen-activated protein kinase pathways in Chinese hamster ovary cells. Endocrinology 1999; 140(3): 1132-40.

[49] Wheeler MB, Lu M, Dillon JS, Leng XH, Chen C, Boyd Iii AE. Functional expression of the rat glucagon-like peptide-I receptor, evidence for coupling to both adenylyl cyclase and phospholipaseC. Endocrinology 1993; 133(1): 57-62.

[50] Perry T, Haughey NJ, Mattson MP, Egan JM, Greig NH. Protection and reversal of excitotoxic neuronal damage by glucagon-like peptide-1 and exendin-4. J Pharmacol Exp Ther 2002; 302(3): 8818.

(C) Chayosumrit et al.; Licensee Bentham Open.

This is an open access article licensed under the terms of the Creative Commons Attribution Non-Commercial License (http://creativecommons.org/licenses/by$\mathrm{nc} / 3.0 /$ ), which permits unrestricted, non-commercial use, distribution and reproduction in any medium, provided the work is properly cited. 